\title{
ASS kann Darmkrebsrisiko senken
}

— Die regelmäßige Einnahme von Acetylsalicylsäure (ASS) senkt das Risiko, an Krebs zu erkranken. Der Effekt geht wesentlich auf die reduzierte Inzidenz von gastrointestinalen und hier vor allem von kolorektalen Tumoren zurück. Die Erkenntnisse zur verminderten Krebsinzidenz bei regelmäßiger ASS-Einnahme gehen auf die Daten der Nurses' Health Study und der Health Professionals Follow-up Study zurück [JAMA Oncol 2016 Mar 3]. Daran hatten sich über 135.000 Frauen und Männer beteiligt, die auch Auskunft über ihren ASS-Konsum gaben.

Wie ein Wissenschaftlerteam um den Seniorautor Andrew Chan vom Massachusetts General Hospital berichtet, erkrankten während der 32-jährigen Nachbeobachtungszeit knapp $21 \%$ der Studienteilnehmer an Krebs. Bei regelmäßiger ASS-Einnahme fiel die Krebsinzidenz insgesamt um 3\% niedriger aus - eine geringe, aber signifikante Reduktion. Zu verdanken war der Effekt vor allem dem Rückgang von gastrointestinalen Malignomen mit einem Minus von $15 \%$. Speziell die Verminderung von kolorektalem Krebs um 19\% schlug hier zu Buche. Als regelmäßig galt eine mindestens zweimal wöchentliche Einnahme von ASS. Der Effekt war dosisabhängig, was für einen kausalen Effekt spricht, ohne ihn freilich zu beweisen. Zu beobachten war die protek-tive Wirkung ab einer Dosis von 162,5-487,5 mg ASS pro Woche, sofern sie sechs bis acht Jahre lang eingenommen wurde. Bei dieser Menge waren 14,0\% weniger kolorektale Karzinome zu verzeichnen. Für 15 oder mehr Tabletten à 325 mg pro Woche lag die Reduktion bei $39,0 \%$. Bezogen auf die Bevölkerung verhinderte der regelmäßige ASS-Konsum laut Aussage

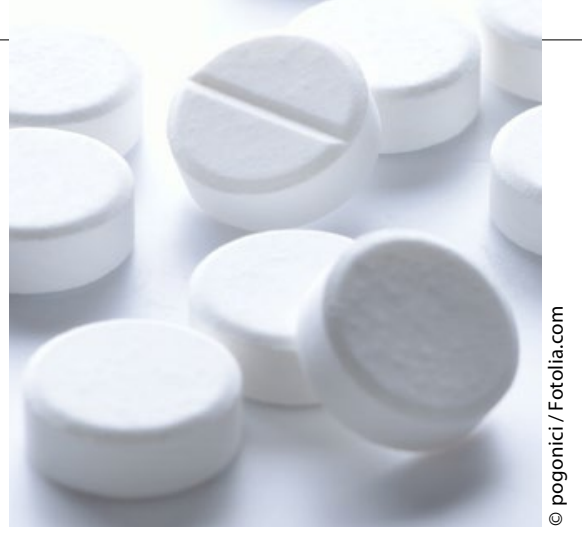

von Chan et al. 1,8\% sämtlicher, $8 \%$ der gastrointestinalen und $10,8 \%$ der kolorektalen bösartigen Tumoren. Mit Blick auf über 50-Jährigen lag die Reduktion der Darmkrebsinzidenz bei $17,0 \%$, sofern die Betreffenden nicht am Koloskopiescreening teilnahmen, und bei $8,5 \%$, sofern sie sich dem Screening unterzogen.

Die Anlässe für die ASS-Einnahme waren im Übrigen nicht ungewöhnlich. Als Gründe gaben die Studienteilnehmer Kopfschmerzen, Arthritis, muskuloskeletale Schmerzen und die Prävention oder Therapie von koronarer Herzkrankheit an.

$r b$

\section{סִ}

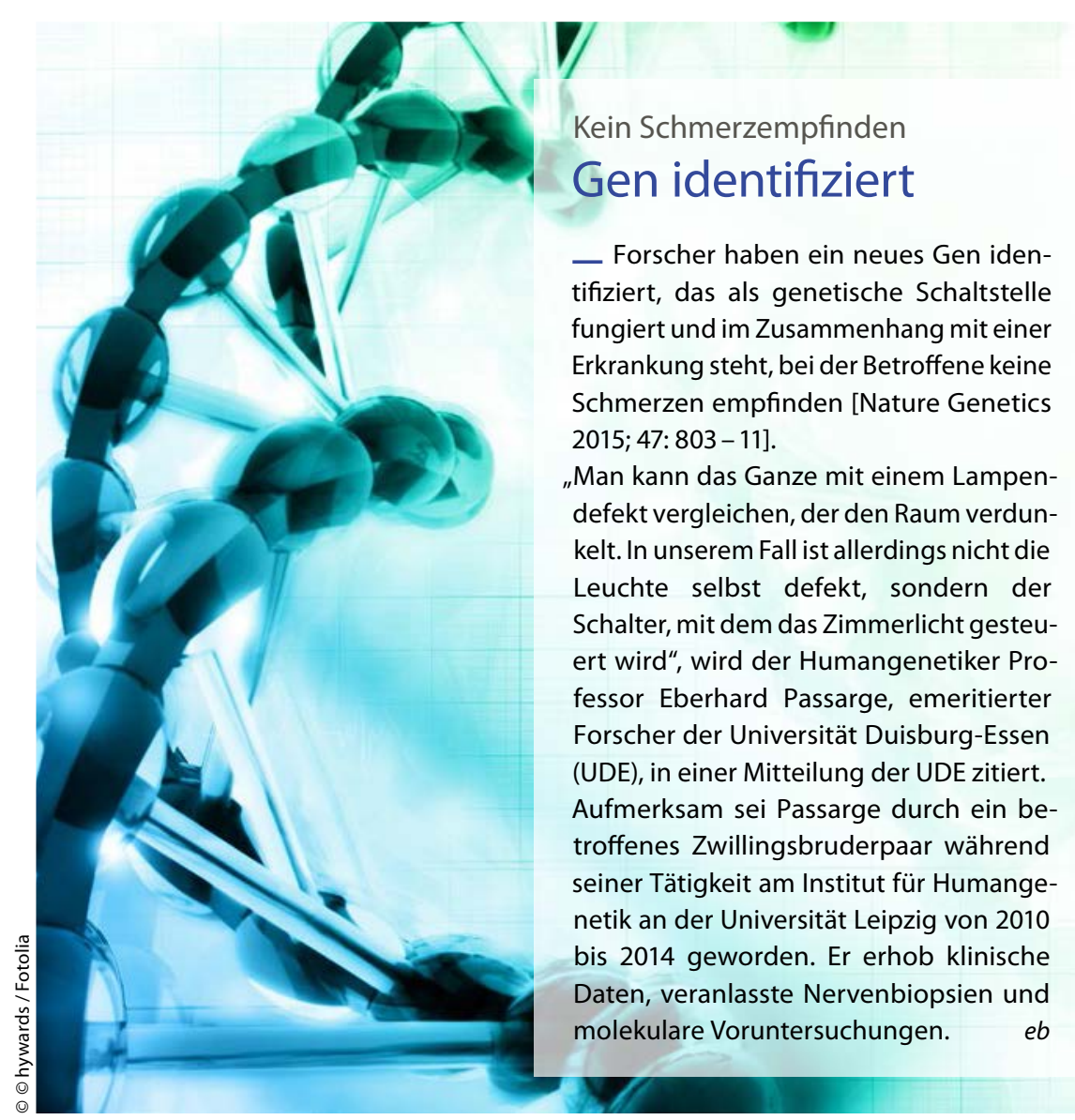

\section{Rückenschmerzen}

\section{Hohe Fehlzeiten in Hessen}

— Rückenbeschwerden haben im vergangenen Jahr bei hessischen Arbeitnehmern für hohe Fehlzeiten gesorgt: Allein wegen Rückenbeschwerden war jeder Erwerbstätige 1,4 Tage arbeitsunfähig. Darauf weist die Techniker Krankenkasse (TK) in Hessen hin und bezieht sich auf erste Daten aus ihrem Gesundheitsreport. Die häufigste Einzeldiagnose unter den Rückenproblemen sind die unspezifischen "Rückenschmerzen", die rund die Hälfte der Beschwerden (0,8 Tage pro Kopf) ausmachen. Damit gehörten Rückenschmerzen mit Atemwegsinfektionen und psychischen Erkrankungen zu den häufigsten Ursachen von Krankschreibungen, heißt es in einer aktuellen Mitteilung der Kasse. Insgesamt waren die bei der TK in Hessen versicherten Erwerbspersonen 2015 15,3 Tage krankgeschrieben.

\section{.}

